\title{
Non-Abelian gauge potentials for ultra-cold atoms with degenerate dark states
}

\author{
J. Ruseckas, ${ }^{1,2}$ G. Juzeliūnas, ${ }^{1}$ P. Öhberg, ${ }^{3}$ and M. Fleischhauer ${ }^{2}$ \\ ${ }^{1}$ Vilnius University Research Institute of Theoretical Physics and Astronomy, \\ A. Goštauto 12, 01108 Vilnius, Lithuania \\ ${ }^{2}$ Fachbereich Physik, Technische Universität Kaiserslautern, \\ D-67663 Kaiserslautern, Germany \\ ${ }^{3}$ Department of Physics, University of Strathclyde, \\ Glasgow G4 ONG, Scotland
}

(Dated: June 27, 2006)

\begin{abstract}
We show that the adiabatic motion of ultra-cold, multi-level atoms in spatially varying laser fields can give rise to effective non-Abelian gauge fields if degenerate adiabatic eigenstates of the atomlaser interaction exist. A pair of such degenerate dark states emerges e.g. if laser fields couple three internal states of an atom to a fourth common one under pairwise two-photon-resonance conditions. For this so-called tripod scheme we derive general conditions for truly non-Abelian gauge potentials and discuss special examples. In particular we show that using orthogonal laser beams with orbital angular momentum an effective magnetic field can be generated that has a monopole component.
\end{abstract}

PACS numbers: 03.65.Vf, 42.50.Gy, 03.75.Lm

Gauge fields are a central building block of the theory of fundamental interactions. As dynamical variables they are responsible for the forces between elementary particles. On the other hand also non-dynamical, i.e. prescribed gauge fields are of interest in a variety of singleand many-body quantum systems. E.g. an external magnetic field applied to a gas of non-interacting electrons can lead to the integer quantum Hall effect [1]. In the presence of a lattice potential, the eigenenergies of the lowest Bloch band form a fractal structure depending on the magnetic flux that passes through the unit cell [2]. If in addition there are strong interactions between the particles as e.g. in a two dimensional electron gas subject to a magnetic field, fractional quantum Hall structures [3] and Laughlin liquids [4] can emerge.

In recent years ultra-cold atomic gases $[5]$ have become an ideal playground to experimentally investigate manybody physics. This is due to their enormous versatility and the advanced experimental techniques available in atomic and optical physics. One of the most fascinating subjects in this context is the study of effects of artificial magnetic fields [6]. To create an artificial magnetic field for neutral atoms one can e.g. rotate the trapping potential confining the atoms. This experimentally feasible but challenging approach is currently pursued in several labs 7]. An alternative is based on the adiabatic motion of $\Lambda$-type 3 -level atoms in laser fields that create a non-degenerate dark state, i.e. an eigenstate of the atom-laser interaction. If the dark-state of the atom is space dependent, the motion of atoms adiabatically following it is associated with a topological or Berry phase [8, 10]. A proper description of such a motion naturally leads to Abelian gauge potentials [9, 10, 11]. As shown in [12, 13] a non-vanishing effective magnetic field can arise e.g. if $\Lambda$-type atoms interact with pairs of laser fields that possess a relative orbital angular momentum.
The advantage of this scheme as compared to rotating traps is that it is not limited to rotationally symmetric configurations. Furthermore in the rotating traps only a constant effective magnetic field is created [], whereas using optical means the effective magnetic field can be controlled and shaped 13. The description of the adiababatic motion of atoms in terms of gauge potentials has been generalized to $j+1 \rightarrow j$ transitions in [14]. The effects of gauge potentials on strongly interacting, bosonic atoms in one-dimensional optical lattices have been analyzed [15], where it was shown that they lead to interesting modifications of the Bose-Hubbard model. An alternative way to create artificial magnetic fields in lattice gases was recently suggested employing laser assisted, state-dependent tunneling [16, 17] or oscillating potentials with spatial modulations [18]. In all of these systems the gauge fields have however $\mathrm{U}(1)$ symmetry, i.e. they are Abelian.

As shown by Wilczek and Zee, non-Abelian gauge fields can arise in the adiabatic dynamics of quantum systems with multiple degenerate eigenstates [19]. One of the interesting properties of non-Abelian gauge potentials is the possibility of magnetic monopoles. The presence of effective magnetic monopole fields in simple quantum systems was first pointed out by Moody, Shapere and Wilczek discussing the adiabatic nuclear rotation in a diatomic molecule [20]. In the present paper we propose an experimentally realizable scheme which allows to study the motion and the interaction of neutral quantum gases in non-Abelian gauge fields. We show in particular that the coupling of multi-level atoms to spatially varying laser fields can give rise to such potentials for the atomic center-of-mass motion. A necessary condition for this is that the atom-laser interaction has degenerate dark eigenstates with a non-vanishing non-adiabatic coupling.

Gauge structures in atomic systems with multiple de- 
generate dark states have first been discussed by Visser and Nienhuis 14 considering atoms with a $j+1 \rightarrow j$ $(j>1)$ transition driven by circularly polarized laser light, as shown in Fig. 1(a). Since in such a scheme the dark states are exactly decoupled, the associated gauge potentials have however again $\mathrm{U}(1)$ symmetry. The simplest system with a non-vanishing adiabatic coupling between degenerate dark states is the so-called tripodscheme shown in Fig. [(b) 21]. For this scheme the possibility of non-Abelian topological phases has been predicted analyzing fractional adiabatic transfer in 22]. Furthermore applications to geometric quantum computation have been put forward and investigated in [23, 24].

After a general discussion of non-Abelian gauge potentials in the adiabatic motion of atoms in laser fields, we will introduce the tripod coupling scheme as the simplest system leading to non-Abelian gauge fields. We then will discuss specific examples. In particular we will show that using orthogonal laser beams with orbital angular momentum an effective magnetic field can be generated that has a monopole component.
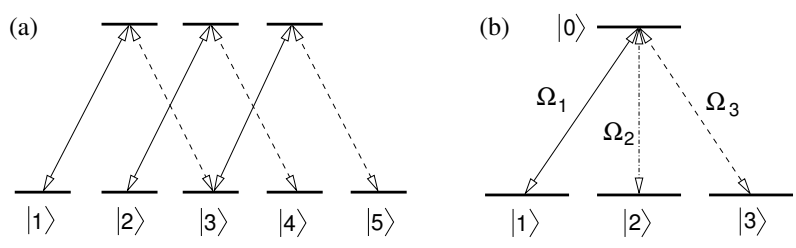

FIG. 1: (a) $j=2 \rightarrow j=1$ transition with two degenerate dark states in the manifolds $\{|1\rangle,|3\rangle,|5\rangle\}$ and $\{|2\rangle,|4\rangle\}$ which are not coupled by non-adiabatic transitions. (b) Tripod coupling scheme forming two degenerate dark states with non-adiabatic coupling.

We start by extending the discussion of Wilczek and Zee [19] and Moody, Shapere and Wilczek [20] to the adiabatic motion of atoms in stationary laser fields. For this we consider atoms with multiple internal states. For fixed position $\mathbf{r}$ the internal Hamiltonian $\hat{H}_{0}(\mathbf{r})$ including the laser interaction can be diagonalized to give a set of say $N$ dressed states $\left|\chi_{n}(\mathbf{r})\right\rangle$ with eigenvalues $\varepsilon_{n}(\mathbf{r})$, where $n=$ $1,2, \ldots, N$. The full quantum state of the atom describing both internal and motional degrees of freedom can then be expanded in terms of the dressed states according to $|\Phi\rangle=\sum_{n=1}^{N} \Psi_{n}(\mathbf{r})\left|\chi_{n}(\mathbf{r})\right\rangle$. The $N$-dimensional column vector of wave-functions $\Psi=\left(\Psi_{1}, \Psi_{2}, \ldots, \Psi_{N}\right)^{\top}$ obeys the Schrödinger equation

$$
i \hbar \frac{\partial}{\partial t} \Psi=\left[\frac{1}{2 m}(-i \hbar \nabla-\mathbf{A})^{2}+V\right] \Psi,
$$

$m$ being the mass of the atoms, and $V$ being an external potential that confines the motion of atoms to a finite region in space. Here $\mathbf{A}$ and $V$ are $N \times N$ matrices appearing due to the position dependence of the atomic dressed states:

$$
\begin{aligned}
\mathbf{A}_{n, m} & =i \hbar\left\langle\chi_{n}(\mathbf{r}) \mid \nabla \chi_{m}(\mathbf{r})\right\rangle, \\
V_{n, m} & =\varepsilon_{n}(\mathbf{r}) \delta_{n, m}+\left\langle\chi_{n}(\mathbf{r})|V(\mathbf{r})| \chi_{m}(\mathbf{r})\right\rangle .
\end{aligned}
$$

The off-diagonal elements of the matrices $\mathbf{A}$ and $V$ are typically much smaller than the difference of the dressed atomic energies. In this case an adiabatic approximation can be applied which amounts to neglecting the offdiagonal contributions. This leads to a separation of the dynamics: Atoms in any one of the dressed states evolve according to a separate Hamiltonian with a $\mathrm{U}(1)$, i.e. Abelian gauge potential.

The adiabatic approximation fails however if there are degenerate (or nearly degenerate) dressed states. This is the case we are interested in. Off-diagonal (nonadiabatic) couplings between the degenerate dressed states can then no longer be ignored. Suppose the first $q$ atomic dressed states are degenerate (or nearly degenerate), and these levels are well separated from the remaining $N-q$. Neglecting transitions to the remaining states, i.e. projecting the full Hamiltonian to this subspace leads to the Schrödinger equation for the reduced column vector $\tilde{\Psi}=\left(\Psi_{1}, \ldots, \Psi_{q}\right)^{\top}$

$$
i \hbar \frac{\partial}{\partial t} \tilde{\Psi}=\left[\frac{1}{2 m}(-i \hbar \nabla-\mathbf{A})^{2}+V+\Phi\right] \tilde{\Psi}
$$

with $\mathbf{A}$ and $V$ being the truncated $q \times q$ matrices. The projection of the term $\mathbf{A}^{2}$ to the $q$ dimensional subspace cannot entirely be expressed in terms of a truncated matrix A. This gives rise to a scalar potential $\Phi$ which is again a $q \times q$ matrix,

$$
\begin{aligned}
\Phi_{n, m} & =\frac{1}{2 m} \sum_{l=q+1}^{N} \mathbf{A}_{n, l} \cdot \mathbf{A}_{l, m} \\
& =\frac{\hbar^{2}}{2 m}\left(\left\langle\nabla \chi_{n} \mid \nabla \chi_{m}\right\rangle+\sum_{k=1}^{q}\left\langle\chi_{n} \mid \nabla \chi_{k}\right\rangle\left\langle\chi_{k} \mid \nabla \chi_{m}\right\rangle\right)
\end{aligned}
$$

with $n, m \in(1, \ldots, q)$. The reduced $q \times q$ matrix $\mathbf{A}$ is called the Berry connection.

Since the adiabatic states $\left|\chi_{1}\right\rangle \ldots\left|\chi_{q}\right\rangle$ are degenerate, any basis generated by a local unitary transformation $U(\mathbf{r})$ within the subspace is equivalent. The corresponding local basis change

$$
\tilde{\Psi} \rightarrow U(\mathbf{r}) \tilde{\Psi}
$$

leads to a transformation of the potentials according to

$$
\begin{aligned}
\mathbf{A} & \rightarrow U(\mathbf{r}) \mathbf{A} U^{\dagger}(\mathbf{r})-i \hbar(\nabla U(\mathbf{r})) U^{\dagger}(\mathbf{r}) \\
\Phi & \rightarrow U(\mathbf{r}) \Phi U^{\dagger}(\mathbf{r}) .
\end{aligned}
$$

These transformation rules show the gauge character of the potentials $\mathbf{A}$ and $\Phi$. 
The Berry connection or vector potential $\mathbf{A}$ is related to a curvature (an effective "magnetic" field) B as:

$$
B_{i}=\frac{1}{2} \epsilon_{i k l} F_{k l}, \quad F_{k l}=\partial_{k} A_{l}-\partial_{l} A_{k}-\frac{i}{\hbar}\left[A_{k}, A_{l}\right] .
$$

Note that the term $\frac{1}{2} \varepsilon_{i k l}\left[A_{k}, A_{l}\right]=(\mathbf{A} \times \mathbf{A})_{i}$ does not vanish in general, since the vector components of $\mathbf{A}$ do not necessarily commute. In fact this term reflects the non-Abelian character of the gauge potentials.

The generalized "magnetic" field transforms under local rotations of the degenerate dressed basis (6) as

$$
\mathbf{B} \rightarrow U(\mathbf{r}) \mathbf{B} U^{\dagger}(\mathbf{r})
$$

Thus, as expected, $\mathbf{B}$ is a true gauge field.

We will now construct a scheme of laser-atom interactions that leads to a $U(2)$ gauge potential. The first requirement is the presence of degenerate (or nearly degenerate) dressed states. Such a condition is fulfilled e.g. for the two systems shown in Fig. 11 Each of them has two degenerate dark states [25], i.e. dressed eigenstates with no component of the excited, radiatively decaying level. Thus the gauge potentials are $2 \times 2$ matrices. In order for them to be truly non-Abelian, the off-diagonal element $i \hbar\left\langle\chi_{1}(\mathbf{r}) \mid \nabla \chi_{2}(\mathbf{r})\right\rangle$ has to be non-zero. One can easily check that this expression always vanishes for the system discussed in 14] and shown in Fig. 11(a). It is nonvanishing however for the so-called tripod scheme shown in Fig. 11 b) 21].

The Hamiltonian of the tripod system reads in interaction representation as

$$
\hat{H}_{0}=-\hbar\left(\Omega_{1}|0\rangle\left\langle 1\left|+\Omega_{2}\right| 0\right\rangle\left\langle 2\left|+\Omega_{3}\right| 0\right\rangle\langle 3|\right)+H . c .,
$$

Parameterizing the Rabi-frequencies $\Omega_{\mu}$ with angle and phase variables according to

$$
\begin{aligned}
& \Omega_{1}=\Omega \sin \theta \cos \phi \mathrm{e}^{i S_{1}} \\
& \Omega_{2}=\Omega \sin \theta \sin \phi \mathrm{e}^{i S_{2}} \\
& \Omega_{3}=\Omega \cos \theta \mathrm{e}^{i S_{3}}
\end{aligned}
$$

where $\Omega=\sqrt{\left|\Omega_{1}\right|^{2}+\left|\Omega_{2}\right|^{2}+\left|\Omega_{3}\right|^{2}}$, the adiabatic dark states read

$$
\begin{aligned}
\left|D_{1}\right\rangle= & \sin \phi \mathrm{e}^{i S_{31}}|1\rangle-\cos \phi \mathrm{e}^{i S_{32}}|2\rangle \\
\left|D_{2}\right\rangle= & \cos \theta \cos \phi \mathrm{e}^{i S_{31}}|1\rangle+\cos \theta \sin \phi \mathrm{e}^{i S_{32}}|2\rangle \\
& -\sin \theta|3\rangle
\end{aligned}
$$

with $S_{i j}=S_{i}-S_{j}$. It is now straight-forward to calculate the vector and scalar gauge potentials. This yields

$$
\begin{aligned}
& \mathbf{A}_{11}=\hbar\left(\cos ^{2} \phi \nabla S_{23}+\sin ^{2} \phi \nabla S_{13}\right), \\
& \mathbf{A}_{12}=\hbar \cos \theta\left(\frac{1}{2} \sin (2 \phi) \nabla S_{12}-i \nabla \phi\right), \\
& \mathbf{A}_{22}=\hbar \cos ^{2} \theta\left(\cos ^{2} \phi \nabla S_{13}+\sin ^{2} \phi \nabla S_{23}\right),
\end{aligned}
$$

and

$$
\begin{aligned}
\Phi_{11} & =\frac{\hbar^{2}}{2 m} \sin ^{2} \theta\left(\frac{1}{4} \sin ^{2}(2 \phi)\left(\nabla S_{12}\right)^{2}+(\nabla \phi)^{2}\right) \\
\Phi_{12} & =\frac{\hbar^{2}}{2 m} \sin \theta\left(\frac{1}{2} \sin (2 \phi) \nabla S_{12}-i \nabla \phi\right) \\
& \left(\frac{1}{2} \sin (2 \theta)\left(\cos ^{2} \phi \nabla S_{13}+\sin ^{2} \phi \nabla S_{23}\right)-i \nabla \theta\right) \\
\Phi_{22} & =\frac{\hbar^{2}}{2 m}\left(\frac{1}{4} \sin ^{2}(2 \theta)\left(\cos ^{2} \phi \nabla S_{13}+\sin ^{2} \phi \nabla S_{23}\right)^{2}\right. \\
& \left.+(\nabla \theta)^{2}\right) .
\end{aligned}
$$

Since the level scheme considered in Fig. 1 corresponds to that of Alkali atoms where $|1\rangle,|2\rangle$, and $|3\rangle$ are Zeeman components of hyperfine levels, it is natural to assume that the external trapping potential is diagonal in these states and has the form $V=V_{1}(\mathbf{r})|1\rangle\left\langle 1\left|+V_{2}(\mathbf{r})\right| 2\right\rangle\langle 2|+$ $V_{3}(\mathbf{r})|3\rangle\langle 3|$. This still takes into account the fact that magnetic, magneto-optical or optical dipole forces can be different in different Zeeman states. According to Eq. (3), the external potential in the adiabatic basis is then given by a $2 \times 2$ matrix with elements $V_{j k}=\left\langle D_{j}|V| D_{k}\right\rangle$. Using the expressions for the dark states (13) and (14), we arrive at

$$
\begin{aligned}
V_{11} & =V_{2} \cos ^{2} \phi+V_{1} \sin ^{2} \phi, \\
V_{12} & =\frac{1}{2}\left(V_{1}-V_{2}\right) \cos \theta \sin (2 \phi), \\
V_{22} & =\left(V_{1} \cos ^{2} \phi+V_{2} \sin ^{2} \phi\right) \cos ^{2} \theta+V_{3} \sin ^{2} \theta .
\end{aligned}
$$

At this point it is instructive to consider some specific examples. Let us first assume that the laser fields coupling levels $|1\rangle$ and $|2\rangle$ are co-propagating, and have the same frequency and the same orbital angular momentum (if any). In this case their relative phase is fixed and can be set $S_{12}=0$. This leads to $S_{13}=S_{23} \equiv S$ and the expressions for the vector potential simplify to

$$
\mathbf{A}=\hbar\left(\begin{array}{cc}
\nabla S & -i \cos \theta \nabla \phi \\
i \cos \theta \nabla \phi & \cos ^{2} \theta \nabla S
\end{array}\right)
$$

The components of the $2 \times 2$ matrix of the effective magnetic field can be easily evaluated and read

$$
\begin{aligned}
\mathbf{B}_{11}= & 0, \\
\mathbf{B}_{12}= & i \hbar \sin \theta \mathrm{e}^{-i S} \nabla \theta \times \nabla \phi \\
& -\hbar \cos \theta \mathrm{e}^{-i S} \nabla S \times \nabla \phi\left(1+\cos ^{2} \theta\right), \\
\mathbf{B}_{22}= & -2 \hbar \cos \theta \sin \theta \nabla \theta \times \nabla S .
\end{aligned}
$$

One recognizes that a large magnetic field requires large gradients of the relative intensities of the fields, parametrized by the angles $\phi$ and $\theta$ and a large gradient of the relative phase $S$. Gradients of $\phi$ and $\theta$ on the order of the wavenumber $k$ can be achieved by using standingwave fields. Large gradients of $S$ can be obtained from 
a running wave $\Omega_{3}$ orthogonal to the other two or by a vortex beam with large orbital angular momentum. In this case magnetic fluxes as large as one (in normalized units) can be reached.

We now construct a specific field configuration that leads to a magnetic monopole. For this let us consider two co-propagating and circularly polarized fields $\Omega_{1,2}$ with opposite orbital angular momenta $\pm \hbar$ along the propagation axis $z$. The field $\Omega_{3}$ propagates in $x$ direction and is linearly polarized along the $y$-axis:

$$
\Omega_{1,2}=\Omega_{0} \frac{\rho}{R} \mathrm{e}^{i(k z \mp \varphi)}, \quad \Omega_{3}=\Omega_{0} \frac{z}{R} \mathrm{e}^{i k^{\prime} x} .
$$

Here $\rho$ is the distance from the $z$-axis and $\varphi$ the azimuthal angle around this axis. It should be noted that these fields have a vanishing divergence and obey the Helmholtz equation. This in contrast to the fields which have been suggested in [26] to create an Abelian magnetic monopole in a $\Lambda$ system. The total intensity of the laser fields (20) vanishes at a origin, which is a singular point.

The vector potential associated with the fields can be calculated from Eq. (15). It reads

$$
\begin{aligned}
& \mathbf{A}=-\hbar \frac{\cos \vartheta}{r \sin \vartheta} \hat{\mathrm{e}}_{\varphi}\left(\begin{array}{ll}
0 & 1 \\
1 & 0
\end{array}\right)+\frac{\hbar}{2}\left(k \hat{\mathrm{e}}_{z}-k^{\prime} \hat{\mathrm{e}}_{x}\right) \times \\
& \times\left[\left(1+\cos ^{2} \vartheta\right)\left(\begin{array}{ll}
1 & 0 \\
0 & 1
\end{array}\right)+\left(1-\cos ^{2} \vartheta\right)\left(\begin{array}{cc}
1 & 0 \\
0 & -1
\end{array}\right)\right] .
\end{aligned}
$$

The first term proportional to $\sigma_{x}$ corresponds to a magnetic monopole of strength one at the origin. This is easily seen by calculating the magnetic field

$$
\mathbf{B}=\frac{\hbar}{r^{2}} \hat{\mathrm{e}}_{r}\left(\begin{array}{ll}
0 & 1 \\
1 & 0
\end{array}\right)+\cdots
$$

The dots indicate non-monopole field contributions proportional to $\sigma_{z}, \sigma_{y}$ and the unity matrix.

In the present paper we have shown that the adiabatic motion of multi-level atoms interacting with spatially varying laser fields in the tripod-coupling configuration can lead to $\mathrm{U}(2)$ non-Abelian gauge potentials. The system can easily be generalized to effective $\mathrm{U}(n)$, $n>2$, gauge structures using atomic configurations with more than three laser fields coupling to a common excited state. The strength of the effective magnetic fields can be large if standing wave configurations or light beams with large orbital angular momentum are used. As a specific example we have identified a configuration of laser fields which leads to a magnetic monopole.

Our approach is complementary to the recent proposal of Osterloh et al. [27], who suggested the generation of effective non-Abelian fields in lattice gases. For this they employed a state-dependent manipulation of tunneling amplitudes by lasers. These proposals make the study of interacting degenerate Bose or Fermi gases in nonAbelian gauge fields experimentally feasible.
M.F. would like to thank R.G. Unanyan for discussions. This work has been supported by the Alexandervon-Humboldt foundation. J.R. has been supported by the EU through the Marie-Curie Trainingssite at the TU Kaiserslautern. P.Ö. wishes to acknowledge the Royal Society of Edinburg for support.

[1] K. v. Klitzing, G. Dorda, and M. Pepper, Phys. Rev. Lett. 45, 494 (1980); Zyun F. Ezawa Quantum Hall Effects, (World Scientific, Singapore, 2000).

[2] D. R. Hofstadter, Phys. Rev. B 14, 2239 (1976).

[3] D. C. Tsui, H. L. Stormer, and A. C. Gossard, Phys. Rev. Lett. 48, 1559 (1982).

[4] R. B. Laughlin, Phys. Rev. Lett. 50, 1395 (1985).

[5] see e.g.: L. Pitaevskii and S. Stringari, Bose-Einstein Condensation (Claredon Press, Oxford 2003).

[6] N. Regnault and Th. Jolicoeur Phys. Rev. Lett. 91, 030402 (2003); Phys. Rev. B 70, 241307(R) (2004).

[7] P. Rosenbusch, D.S. Petrov, S. Sinha, F. Chevy, V. Bretin, Y. Castin, G. Shlyapnikov, and J. Dalibard Phys. Rev. Lett. 88, 250403 (2002); V. Schweikhard, I. Coddington, P. Engels, V. P. Mogendorff, and E. A. Cornell, Phys. Rev. Lett. 92, 040404 (2004); V. Bretin, S. Stock, Y. Seurin, and J. Dalibard, Phys. Rev. Lett. 92, 050403 (2004).

[8] M. V. Berry, Proc. Roy. Soc. London A 392, 45 (1984).

[9] R. Jackiw, Adv. At. Mol. Phys. 21, 71 (1988).

[10] A. Shapere and F. Wilczek (eds.), Geometric Phases in Physics (World Scientific, Singapore, 1989).

[11] R. Dum and M. Olshanii, Phys. Rev. Lett. 76, 1788 (1996).

[12] G. Juzeliunas and P. Öhberg, Phys. Rev. Lett. 93, 033602 (2004).

[13] G. Juzeliunas, P. Öhberg, J. Ruseckas, and A. Klein, preprint cond-mat/0412015.

[14] P. M. Visser and G. Nienhuis, Phys. Rev. A 57, 4581 (1998).

[15] K. V. Krutitsky and R. Graham, Phys. Rev. Lett. 91, 240406 (2003).

[16] D. Jaksch and P. Zoller, New J. Phys. 5, 56 (2003).

[17] E. Mueller, Phys. Rev. A 70, 041603 (R) (2004).

[18] A.S. Sørensen, E. Demler, and M. D. Lukin, Phys. Rev. Lett. 94, 086803 (2005).

[19] F. Wilczek and A. Zee, Phys. Rev. Lett. 52, 2111 (1984).

[20] J. Moody, A. Shapere, and F. Wilczek, Phys. Rev. Lett. 56, 893 (1986).

[21] R. G. Unanyan, M. Fleischhauer, B. E. Shore, and K. Bergmann, Opt. Commun. 155, 144 (1998).

[22] R. G. Unanyan, B. W. Shore, and K. Bergmann, Phys. Rev. A 59, 2910 (1999).

[23] L.-M. Duan, J. I. Cirac, and P. Zoller, Science 292, 1695 (2001).

[24] R. G. Unanyan and M. Fleischhauer, Phys. Rev. A 69, $050302(\mathrm{R})$ (2004).

[25] see E. Arimondo, Progress in Optics 35, (1996).

[26] P. Zhang, Y. Li, and C.P. Sun, preprint quant$\mathrm{ph} / 04040108$.

[27] K. Osterloh, M. Baig, L. Santos, P. Zoller, and M. Lewenstein, preprint cond-mat/0502251. 